\title{
ADVANCED DISTRIBUTED CLOUD SHARING IN SMART TV
}

\author{
Raghuveeraachari.K \\ ${ }^{\text {I} S t u d e n t-(M . T e c h .), ~ C S E ~ D e p t ., ~ B V R I T ~ C o l l e g e, ~ A . P ., ~ I N D I A ~}$
}

\begin{abstract}
Advanced Distributed Cloud Sharing in SMART TV is an embedded based integrated application with the advanced directory distribution system, it is a built-in application on SMART Television. The Smart TV distributed directory script which includes the different customizable and advanced features with the single press button. The cloud distribution system is studied to design the unique functionality in order to satisfy the greatest number of the Smart TV users in worldwide. Smart cloud application with the directory allows the users to create the files and the cloud directories on the smart cloud network by without prompting the URL link for submission on the local application. With the smart cloud app advanced features the integrated directory is not that simple to linkup the cloud directories with the other devices. Therefore, the smart app users will be to create the multiple types of cloud directories on the vendor cloud network. Cloud Smart television app developers can also create the custom functionalities in order to organize and synchronize the unique user cloud directories within seconds. The vendor or manufacturer Smart Cloud Service allows the television users to backup the smartapp user data and to synchronize the same data to the multiple devices which is connected to the cloud service on the network. User smart device will be automatically detected on the vendor cloud.
\end{abstract}

Whereas, the smart cloud users can also restore the user data on to the another smart device which supports the Vendor Cloud service with full access and smart user can also restore the current smart device data after the function and operation of restore method performed by the smart device user and user can also reset the data at any time. $* * *$

\section{INTRODUCTION}

Advanced Distributed based Cloud Network Sharing oriented SMART Television is an innovative software product in embedded systems with the unique and the smart distinctive application for the Smart Television path. Smart distributive cloud with the unique process with the distributive management system This embedded application is being used by the many international TV brands which can hit up with the new innovative ideas.

Application can also be developed with the SDK's for the development of the innovative Smart Television with the Smart Cloud features with integrated and smart app built into the Smart TV as a firmware. This innovative technology adds the new milestones into the smart cloud app development with the new evolution.

Cloud directory offers with the many different features with advanced techniques which allows the embedded application with more stability, which provides the in depth security, with the fast performance with dual core processor which embedded into the Smart TV, which is introduced with the new and effective advanced features into it. Smart Cloud Television Application mainly handles the multiple requests received from the various smart devices on the same network, which it allows the new functionalities and operations to perform with powerful operations with the more advanced functionality with the most basic in to the smart television.
It uses the smart engine for handling the smart applications and other smart script which is embedded into integrated electronic memory chip, application also supports with the MVC architecture with internet browsing capabilities with the coupled browsing add-ons with object oriented methods involved in it.

It makes the use of jQuery in integrated web browser of smart television which enriches the smart television user interface with the advanced enhancements. The database complements is used which can finalize the smart user choices with the various advanced multiple technologies.

Cloud directory distribution in smart television is a fully featured cloud based smart directory embedded software which can be utilized as the advanced integrated addition of technologies in to the smart device as the smart standalone device.

\section{CONCEPT OF ADVANCED CLOUD}

DIRECTORY DISTRIBUTED SHARING IN

\section{SMART TV}

- Advanced Distributed Directory Sharing in Cloud SMART TV is a directory and file sharing via the cloud network of the particular television manufacturer or vendor. Cloud based Smart TV uses the features of secure sharing of the media links in the cloud connected network. 
- Accessibility of permission grants of the cloud folders and the cloud files is enabled with the folder publishing technique on the smart TV memory. These cloud data of television can be accessed using the different modern web browsers for browsing the pages of cloud data and for synchronizing the television integrated data on cloud.

- Navigation on the television is very easy to operate such as by using drag and the drop operations in smart television from smart tv dashboard. The file operations on smart television such as viewing of files of different extensions on smart cloud through the remote network.

- The current system enables to connect the smart television network to the cloud directory and it uses the same for distributions on the smart cloud network. The cloud television directory distribution system is highly scalable with advanced security of the smart tv applications on the cloud.

- The smart tv with the different features also changes the viewer experience to outstanding levels, it also delivers the exceptional remote access to the vendor cloud. It is packed up with the advanced features with the robust cloud panel with the directory cloud connectivity.

- Smart Panel with the unique secret keys for tv panel. Smart TV cloud has the quality of service with enhancements. By using the smart television cloud application software the smart user portal website is accessed by achieving the desired smart access point network.

- Smart Cloud TV takes the outstanding positions in worldwide with the most popular features. Smart TV cloud software is an open license based open cloud directory sharing platform for smart users.

- It is more simple and compatible with the mobile applications. Smart cloud can be hosted more securely on the vendor smart servers and smart clustered clouds. More easy that smart user can use the television embedded software.

- It is designed according to the smart television needs and requirements to provide the high class security to the clustered cloud servers to control independently.

- Smart Cloud administrators can easily install the embedded application in the smart television which easily connects spontaneously with the existing cloud directories and also supports the smart cloud user's external storage devices without any upgradation.

\section{ADVANCED ARCHITECTURE}

\section{Clustered Smart Cloud Network}

Advanced Directory Distribution

\section{Smart Television: Embedded Memory}

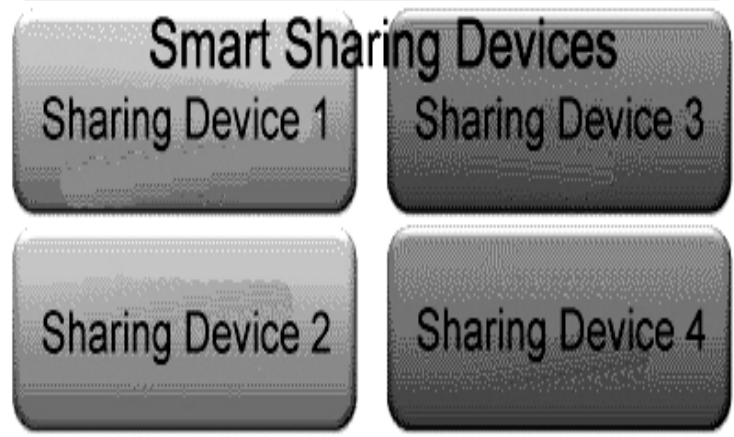

Fig-1: Architecture for Advanced Distributed Cloud Sharing in SMART TV

\subsection{Process}

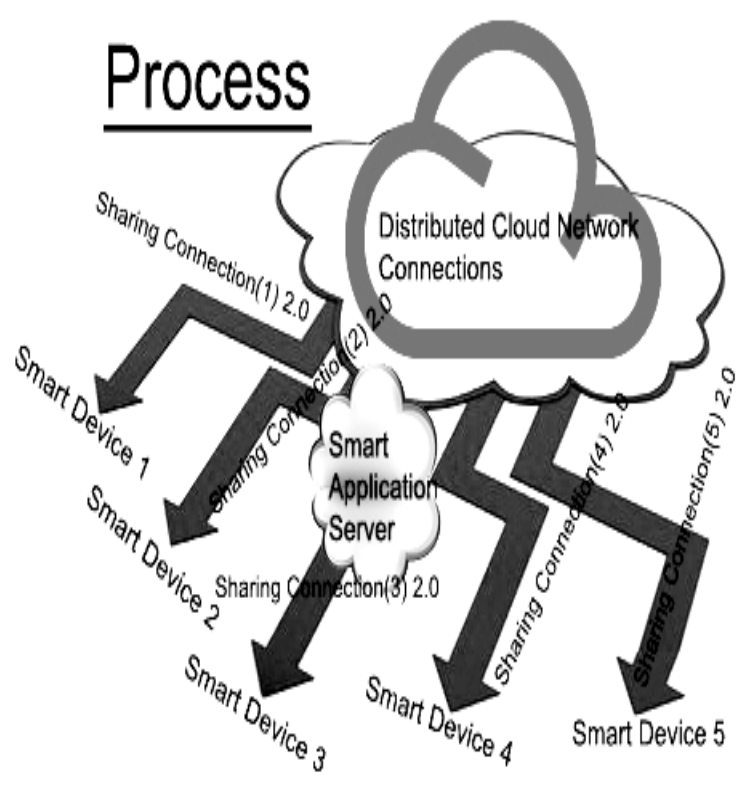

Fig-2: Process of Advanced Distributed Cloud Sharing in SMART TV 


\section{CONCLUSIONS}

This paper shows that how the distributive cloud sharing works with the smart devices on the smart cloud network such as Smart Televisions. Multi Cloud sharing Television has the very huge interface which involves with the large database connections with the multiple hub on the cloud port in order to approach the smart appliances on the remote network through the wireless channel or connectivity in the distributed network system which it allows the smart device or television customers to scroll and enter into the distributive cloud dashboard with home cloud screen with different options and various categories displays on the television screen. On switching into the smart television set the smart cloud application automatically arrives on the television screen with balloon hints and dialogue messages on the screen. Smart apps user will get the ultimate access to the menu with more helpful information displayed on the screen with detailed menu information with instructions on the screen. The smart television integrated software automatically detects the user remote network along with the status of the connectivity. It uses the potential firmware for the smart tv upgradation.

I hereby conclude that distribution based cloud private data sharing is possible with the smart television sets with the cloud vendor user accounts with cloud credentials support. This type of feature is very much helpful to the smart based television set users.

\section{REFERENCES}

[1]. WangWenqiang, AnwitamanDatta, TienTuanAnhDinh, "CityontheSky: Extending XACMLfor Flexible, Secure Data Sharing on the Cloud" year: 2012, Vol.10, pp151-172.

[2]. R.Karthikeyan, B.ChandraMohan, K.S.Arulmozhi, "Optimizing Resource Sharing in Cloud Computing" Published: 2011, pp50to55.

[3]. AimiliaTasidou, PavlosS.Efraimidis, GeorgeDrosatos, "Privacy-Preserving Television Audience Measurement Using Smart TVs "year: 2012, Volume376, pp223-234. 